\title{
Forward: A new kind of endoscopists for advanced therapeutic endoscopy
}

\author{
Jean-François Rey \\ Hepato-Gastroenterology Department, Institut Arnault Tzanck, St. Laurent du Var 06700, France. \\ Correspondence to: Prof. Jean-Francois Rey, Hepato-Gastroenterology Department, Institut Arnault Tzanck, St. Laurent du Var \\ 06700, France. E-mail: jean-francois.rey@orange.fr
}

How to cite this article: Rey JF. Forward: A new kind of endoscopists for advanced therapeutic endoscopy. Mini-invasive Surg 2021;5:21. https://dx.doi.org/10.20517/2574-1225.2021.14

Received: 4 Feb 2021 Accepted: 7 Feb 2021 Published: 8 May 2021

Academic Editor: Giulio Belli Copy Editor: Xi-Jun Chen Production Editor: Xi-Jun Chen

Since the first endoscopic papillotomy carried out by the physician M. Classen in 1993 (München, Germany, simultaneously with K. Kawai, Kyoto, Japan), numerous non-invasive procedures have been performed by physicians and surgeons with different backgrounds in the biliary-pancreatography field, as well as in all parts of digestive endoscopy. In 2007, natural orifice transluminal endoscopic surgery (NOTES) was more experimental than a promising routine procedure, but it led to the development of devices for full-thickness resection (FTRD), endoscopic submucosal dissection (ESD), and stenting procedures to avoid aggressive palliative surgery. Development of therapeutic endoscopic ultrasonography (EUS) during the last decade has allowed the bile duct access to drainage even when traditional endoscopic retrograde cholangiopancreatography (EPRC) is impossible due to duodenal obstruction. Depending on local teaching organization, all of these therapeutic procedures are performed by either physicians or surgeons. The Chinese Endoscopic Society has proposed the global concept of Endoscopology Society with a special interest for some advanced procedures including in the area called Super Minimal Invasive Surgery (SMIS).

This trend leads to the differentiation as well as reunification of various types of endoscopists. They differentiate when only about $10 \%$ of physicians are accredited to perform these very skillful therapeutic procedures, with the other $90 \%$ preforming gastroscopy and colonoscopy Level 1 (dilation, variceal ligation, polypectomy, etc.). For example, they should not perform ERCP without a very demanding initial training as well as regular practice. ERCP can be carried out in any endoscopy unit, as was done in the early 1980s, 
because it is no less acceptable for patient safety. SMIS, large ESD, full-thickness resection, and stenting procedures can reunify physicians and surgeons in the same crucial but narrow field even if they have different backgrounds.

Consequently, we should organize common training to qualify endoscopists who carry out SMIS and all advanced therapeutic procedures. They should have access to high-level qualification as provided by Research Institute against Digestive Cancer (IRCAD) to avoid malpractice due to inadequate training. When we started ERCP in the 1970s, it was on our own as we did not have a teacher; we were innovators. This is not acceptable in the 21 st century. However, common high-level diagnosis endoscopy without minute teaching should not be a pathway for poor surgical colleagues to perform poor diagnosis procedures. With high-resolution endoscopes, imaging-enhanced endoscopy, daily use of classifications for characterization, and diagnosis endoscopy also require a skillful experience and should not be considered as an accessory to a limited surgical practice. For our patient safety, the best is both trained and skillful (surgeons or physicians) endoscopists for high-level therapeutic endoscopy and trained and skillful physicians or surgeons for diagnosis endoscopy.

This new concept needs resources: diagnosis endoscopy requires fewer resources than high-level therapeutic endoscopy, which needs multiple expensive devices. In endoscopy units, nearly all diagnostic endoscopic procedures are carried out in clinics, while, for advanced therapeutic procedures, patients usually have to stay some hours to days in the hospital.

In this special issue focused on biliary and pancreatic diseases, we review all possibilities of advanced diagnosis or therapeutic procedures described by world experts based on clinical practice for the best management of our patients. High-quality endoscopy is mandatory due to its importance in global healthcare.

\section{DECLARATIONS}

Authors' contributions

The author contributed solely to the article.

\section{Availability of data and materials}

Not applicable.

\section{Financial support and sponsorship}

None.

\section{Conflicts of interest}

The author declared that there are no conflicts of interest.

\section{Ethical approval and consent to participate}

Not applicable.

\section{Consent for publication}

Not applicable.

\section{Copyright}

(c) The Author(s) 2021. 
